Neurosurg Focus 8 (6):Introduction, 2000, Click here to return to Table of Contents

\title{
Introduction to odontoid fractures: controversies in the management of odontoid fractures
}

\author{
Gregory J. Przybylski, M.D. \\ Department of Neurosurgery, Jefferson Medical College, Philadelphia, Pennsylvania
}

\begin{abstract}
Although odontoid fractures were first classified more than 25 years ago,${ }^{5}$ the management of these fractures remains controversial. Whereas the nonoperative management with external immobilization devices often leads to successful healing, certain fracture features and patient characteristics have been associated with an increased risk of bone nonunion. ${ }^{6,17}$ Recent technological advances in cervical spine instrumentation techniques have provided additional options for the management of these common fractures. ${ }^{1,12,13,24,27,28}$ However, an absence of rigorous studies in which the investigators compare management methods may contribute to the varying methods used by spine surgeons when approaching similar injuries.

In this issue of Neurosurgical Focus, various aspects of the management of odontoid fractures are examined. In an evidence-based analysis of methods for treating odontoid fractures the authors examine the best available published data to determine the level of scientific data supporting the use of various treatment options. ${ }^{3}$ In epidemiological studies the investigators examine factors contributing to the unusual incidence of spinal cord injury with odontoid fractures as well as the unique considerations that may be attendant on the management of posteriorly displaced fractures. In two other studies the authors discuss the application of specific surgical techniques in treating odontoid fractures, whereas in an additional two reports the authors evaluate the difficulties in managing elderly patients with these injuries. Finally, the use of newer osteoinductive agents is examined as a potential adjunct in the surgical management of bony defects in the dens.
\end{abstract}

In this introduction, the epidemiology of odontoid fractures as well as imaging techniques and treatment options are reviewed. The papers in this issue are also reviewed for key points. Finally, some recommendations for future studies are provided.

\section{EPIDEMIOLOGY OF ODONTOID FRACTURES}

Odontoid fractures commonly occur as a result of traumatic cervical spine injuries, and they represent nearly $10 \%$ of all cervical fractures. ${ }^{17}$ Although these fractures occur in all age groups, younger patients often sustain these injuries after motor vehicle accidents, whereas older patients often sustain odontoid fractures after a fall. Whereas most patients complain of neck pain, neurological injuries are unusual. Significant neurological injuries can include monoparesis and quadriplegia and are usually associated with fractures through the odontoid base. ${ }^{21}$

These fractures were classified into three distinct types by Anderson and D'Alonzo ${ }^{5}$ in 1974 . Type I odontoid fractures are quite uncommon and involve an avulsion of the rostral-most portion of the dens. In contrast, Type II odontoid fractures represent approximately two thirds of all odontoid fractures. In a study of the morphometry of the dens the authors demonstrated a reduction in cortical thickness, trabecular bone volume, and trabecular lattice formation at the base of the dens, which may contribute to the frequency of this fracture type. ${ }^{4}$ The fracture line traverses the base of the odontoid process at the junction of the vertebral body. A lateral bending force has been pro- posed as the mechanism causing the development of Type II fractures. ${ }^{20}$ Finally, Type III fractures actually extend into the body of the axis, frequently involving the superior articular surface. These fractures may be caused by a flexion moment through the occiput. ${ }^{20}$ In more than half of the patients with these lesions displaced fractures are revealed on the initial cervical radiograph. Anterior displacements are most common and are seen in $66 \%$ of patients with displaced Type II fractures and $82 \%$ of those with displaced Type III fractures. ${ }^{17}$

\section{IMAGING OF ODONTOID FRACTURES}

Plain radiography remains the mainstay for identification of odontoid fractures. ${ }^{2,10}$ Both lateral-view and openmouth view radiography may demonstrate the fracture. The magnitude and direction of displacement should be noted because this may be related to failed bone union when nonoperative management is undertaken. In addition, the ligamentous stability of the atlantoaxial joint can be estimated by measuring the atlantodental interval. Fielding, et al., ${ }^{13}$ have suggested that a greater than $3-\mathrm{mm}$ separation between the anterior $\mathrm{C}-1$ ring and the dens implies possible transverse ligament disruption. In addition, Spence, et al., ${ }^{26}$ have suggested that when the lateral masses of the atlas overhang the superior articular facets of the axis by more than $7 \mathrm{~mm}$, injury of this ligament may similarly occur.

The use of CT scanning may be quite helpful in demon- 
strating the plane of the fracture line as well as the degree of comminution. ${ }^{10}$ Both sagittal and coronal CT reconstructions often delineate the fractures more precisely than plain radiographs. However, CT scanning occasionally fails to demonstrate a fracture in the transverse plane if the gantry angle is parallel to the fracture line.

Magnetic resonance imaging has a more limited role in evaluating patients who have sustained odontoid fractures without neurological injuries. However, evaluation of the integrity of the transverse ligament can be facilitated using magnetic resonance imaging. ${ }^{16}$ Multiplanar gradient echo modalities have successfully demonstrated disruption of this ligament, prompting operative treatment rather than applications of external immobilization devices alone.

\section{TREATMENT OPTIONS}

A variety of external immobilization techniques and operative procedures has been described in the management of odontoid fractures. Recognition of the fracture type is imperative, because the success of different techniques varies depending on fracture type. The spine surgeon should be familiar with a variety of management techniques to tailor treatment to the individual situation.

Because Type I fractures are quite uncommon, it is difficult to compare the various treatment methods. In most reports the authors describe the use of external immobilization therapy in which either a rigid cervical collar or a halo vest is worn, and successful bone union is reported to occur in most patients. However, there have been reports on cases of unstable Type I fractures requiring internal fixation.

Numerous methods have been described for the management of the more common Type II fractures. ${ }^{8,9,17,22,25}$ Overall, a nonunion rate of more than one third has been described when the various treatment modalities have been used. This has prompted evaluation of factors associated with nonunion that may help identify optimum treatment strategies for certain characteristics. These have included age older than 40 years, larger-magnitude displacements $(>4-6 \mathrm{~mm})$, and posteriorly displaced fractures. ${ }^{6,17}$ However, some of these factors have not been consistently associated with failed bone union. The management of these fractures in the elderly has been particularly challenging. ${ }^{18,23}$

Although external immobilization has been frequently applied in the treatment of Type II fractures, the use of less rigid devices such as hard cervical collars and Minerva jackets is associated with nonunion more commonly than with halo vest immobilization. ${ }^{11,19}$ Despite the greater degree of immobilization conferred by the halo device when compared with other external devices, however, the rate of fusion failure is still higher than when surgical techniques are undertaken.

A posterior atlantoaxial fusion procedure in which instrumentation is placed has been a common surgical method for treating odontoid fractures. Although the use of this treatment does not directly address the fracture location, stability of the atlantoaxial joint is achieved, and the rate of fusion is high. The Brooks technique for placing bone between the atlas and axis, rather than dorsal to it, seems to facilitate successful bone union. ${ }^{7}$ However, the technique has been associated with frequent nonunion in the treatment of atlantoaxial instability in patients with rheu- matoid arthritis and os odontoideum. This may have prompted the evolution of other posterior fixation techniques such as transarticular screw fixation. ${ }^{15,26}$ Although this technique requires neuroimaging assistance and entails additional risks of vertebral artery injury, incremental improvements in fusion rates have been reported, particularly in patients at higher risk for pseudathrosis. Preoperative imaging studies to identify the location of the vertebral artery and the size of the isthmus of the axis are imperative to determine the safety of placing transarticular screws. Finally, direct fracture reapproximation has been achieved by performing odontoid screw fixation via the anterior approach. ${ }^{1,12,14,15,27}$ Advocates of this technique suggest that fusion rates are quite high and that atlantoaxial rotation is maintained as compared with poorer results obtained using posterior techniques. The results of biomechanical studies suggest that acceptable stabilization is provided by a single-screw technique. ${ }^{24}$ However, the fracture configuration may preclude successful anterior screw fixation. Sufficient preservation of the anteroinferior portion of the $\mathrm{C}-2$ body is needed to provide resistance to screw displacement.

Although operative as opposed to nonoperative therapies have yielded better outcomes in patients with Type II fractures, most Type III fractures are successfully healed after the patient undergoes halo vest immobilization. ${ }^{8,9,11}$ Other external devices do not provide a similar rate of fusion. It has been suggested that the larger surface area of exposed bone facilitates union without requiring supplemental internal fixation.

\section{Synopsis Of Papers In This Issue}

In an evidence-based analysis of treatment techniques for odontoid fractures the authors discuss the limited guidance provided by the best available peer-reviewed publications. Although nearly 100 articles published over 30 years were reviewed, only one third met the selection criteria for evidence-based analysis. Moreover, none of the articles included Class I or Class II evidence. As a result, only treatment options can be found in the current literature, and no conclusive treatment standards or guidelines could be determined. Whereas the studies reviewed support the use of external immobilization in the management of Type I and Type III fractures, both external immobilization and surgical therapeutical options were identified as treatment options for Type II fractures.

The growing evidence for the efficacy of anterior odontoid screw fixation is demonstrated in the article written by Apfelbaum, et al., in which they describe the success and limitations of this technique in a large series of patients. They found a high rate of union regardless of patient age or the magnitude and direction of fracture displacement. The technique was found to be successful in patients in whom surgery was performed up to 6 months postfracture. However, the fracture obliquity was related to the success of this method. In addition, screw-related complications (screw pullout and screw backout) occurred in $11 \%$ of cases. An excellent presentation of the surgical technique is provided.

Other surgical methods for managing odontoid fractures are described in a technique-and-complication format in the third article. A discussion of the selection criteria for each method as well as the limitations encountered 
may assist the spine surgeon in choosing the best method when surgery is warranted.

Although neurological- and respiratory-related complications are not commonly associated with odontoid fractures, this issue contains two articles in which the authors discuss the epidemiology of spinal cord injury in odontoid fractures. The fracture displacement and spinal canal size are identified as factors associated with risk of neurological injury. Whereas posteriorly displaced fractures have been more commonly associated with nonunion after external immobilization therapy, the risk of acute respiratory failure during surgical reduction of these fractures has only been described anecdotally. A frequent incidence of respiratory distress associated with possible death is presented in the setting of cervical traction for the reduction of posteriorly displaced fractures, and the authors offer recommendations for the avoidance of this complication.

Special concerns have been raised regarding the management of elderly patients with odontoid fractures. Although comorbidities occasionally limit the practice of surgical stabilization techniques, osteopenia may also limit the success of placing fixation devices. In two articles the authors discuss different approaches to the management of the elderly patient with odontoid fractures. Despite concerns over surgery-related deaths and instrumentation failure, the authors suggest that advanced age alone should not preclude consideration of surgical stabilization in patients with fractures at high risk of nonunion.

In the final article the investigators explore the use of osteoinductive agents as a supplemental treatment of odontoid fractures. There has been a growing interest in understanding the biological features of bone healing and taking advantage of chemical messengers to stimulate osteoblast activity. These methods may eventually play a role in managing comminuted fractures or unreducible fracture gaps, particularly in older patients.

\section{Future Considerations in Odontoid Fracture Management}

The evidence-based analysis of the current literature on the management of odontoid fractures demonstrates the limitations of retrospective reviews of single management methods in providing guidance to spine surgeons in terms of the best treatment available for a fairly common injury. Certainly an effort to develop a multicenter investigation in which investigators compare the fusion rate of halo immobilization (likely the best external device) with that of standard operative technique in the management of Type II odontoid fractures would help provide a clearer indication for the best treatment of this condition. Moreover, future investigations into the manipulation of bone growth factors may facilitate the use of less invasive methods of treating fracture types associated with frequent nonunion.

\section{References}

1. Aebi M, Etter C, Coscia M: Fractures of the odontoid process. Treatment with anterior screw fixation. Spine 14:1065-1070, 1989.

2. Althoff B: Fracture of the odontoid process. A clinical and radiographic study. Acta Orthop Scand Suppl 177:1-95, 1979.

3. American Medical Assocation: Attributes to Guide the Development and Evaluation of Practice Parameters/Guidelines. Chicago: American Medical Association, 1996
4. Amling M, Posl M, Wening VJ, et al: Structural heterogeneity within the axis: the main cause in the etiology of dens fractures. A histomorphometric analysis of 37 normal and osteoporotic autopsy cases. J Neurosurg 83:330-335, 1995

5. Anderson LD, D'Alonzo RT: Fractures of the odontoid process of the axis. J Bone Joint Surg (Am) 56:1663-1674,1978

6. Apuzzo MLJ, Heiden JS, Weiss MH, et al: Acute fractures of odontoid process. An analysis of 45 cases. J Neurosurg 48: 85-91, 1978

7. Brooks AL, Jenkins EB: Atlanto-axial arthrodesis by the wedge compression method. J Bone Joint Surg (Am) 60:279-284, 1978

8. Clark CR, White AA III: Fractures of the dens. A multicenter study. J Bone Joint Surg (Am) 67:1340-1348, 1985

9. Dunn ME, Seljeskog EL: Experience in the management of odontoid process injuries: an analysis of 128 cases. Neurosurgery 18:306-310, 1986

10. Ehara S, el-Khoury GY, Clark CR: Radiologic evaluation of dens fracture. Role of plain radiography and tomography. Spine 17:475-479, 1992

11. Ekong CE, Schwartz ML, Tator $\mathrm{CH}$, et al: Odontoid fracture: management with early immobilization using the halo device. Neurosurgery 9:631-637, 1981

12. Etter C, Coscia M, Jaberg H, et al: Direct anterior fixation of dens with a cannulated screw system. Spine 16:S25-S32, 1991

13. Fielding JW, Cochran GVB, Lawsing JF III, et al: Tears of the transverse ligament of the atlas: a clinical and biomechanical study. J Bone Joint Surg (Am) 56:1683-1691, 1974

14. Geisler FH, Cheng C, Poka A, et al: Anterior screw fixation of posteriorly displaced Type II odontoid fractures. Neurosurgery 25:30-38, 1989

15. Graziano G, Jaggers C, Lee M, et al: A comparative study of fixation techniques for Type II fractures of the odontoid process. Spine 18:2383-2387, 1993

16. Greene KA, Dickman CA, Marciano FF, et al: Transverse atlantal ligament disruption associated with odontoid fractures. Spine 19:2307-2314, 1994

17. Hadley MN, Browner C, Sonntag VKH: Axis fractures: a comprehensive review of management and treatment in 107 cases. Neurosurgery 17:281-290, 1985

18. Hanigan WC, Powell FC, Elwood PW, et al: Odontoid fractures in elderly patients. J Neurosurg 78:32-35, 1993

19. Maiman DJ, Larson SJ: Management of odontoid fractures. Neurosurgery 11:471-476, 1982

20. Mouradian WH, Fietti VG Jr, Cochran GV, et al: Fractures of the odontoid: a laboratory and clinical study of mechanisms. Orthop Clin North Am 9:985-1001, 1978

21. Przybylski GJ, Welch WC: Longitudinal atlantoaxial dislocation with type III odontoid fracture. Case report and review of literature. J Neurosurg 84:666-670, 1996

22. Ryan MD, Taylor TFK: Odontoid fractures. A rational approach to treatment. J Bone Joint Surg (Br) 64:416-421, 1982

23. Ryan MD, Taylor TFK: Odontoid fractures in the elderly. J Spinal Dis 6:397-401, 1993

24. Sasso R, Doherty BJ, Crawford MJ, et al: Biomechanics of odontoid fracture fixation. Comparison of the one- and twoscrew technique. Spine 18: 1950-1953, 1993

25. Schiess RJ, DeSaussure RL, Robertson JT: Choice of treatment of odontoid fracture. J Neurosurg 57:496-499, 1982

26. Spence KF Jr, Decker S, sell KW: Bursting atlantal fracture associated with rupture of the transverse ligament. J Bone Joint Surg (Am) 52:543-49, 1970

27. Stillerman CB, Wilson JA: Atlanto-axial stabilization with posterior transarticular screw fixation: technical description and report of 22 cases. Neurosurgery 32:948-955, 1993

28. Subach BR, Morone MA, Haid RW Jr, et al: Management of acute fractures with single-screw anterior fixation. Neurosurgery 45:812-820, 1999 